\title{
Impact of early life stress on susceptibility to chronic social stress in adult mice
}

\author{
A. Lepeshko ${ }^{1,2 *}$, V. Reshetnikov ${ }^{1}$, N. Bondar ${ }^{1,2}$ \\ ${ }^{1}$ Institute of Cytology and Genetics SB RAS, Novosibirsk, Russia \\ ${ }^{2}$ Novosibirsk State University, Novosibirsk, Russia \\ *e-mail: arinal@bionet.nsc.ru
}

Key words: maternal separation, social defeat stress, behavioural tests

Motivation and Aim: Despite the growing population of depressed patients and development of new antidepressants, fewer than half achieve full remission and many are not responsive with currently available treatments, including antidepressant medications and psychotherapies. One of the possible reasons for this situation is our incomplete understanding of the mechanisms of the depression and the wide individual variations seen in response to prolonged stress, an important risk factor for depression. A significant contribution to the individual diversities in response to stress can be caused due to previous stress experience, in particular, the adverse experience in childhood. The aim of this study is investigation of early life stress influence on susceptibility to social stress in adulthood.

Methods and Algorithms: As a model of early life stress we used prolonged repeated maternal separation (MS) for two first weeks of life. Further, in adult life chronic social defeat stress paradigm (SS) during 10 days was used. Thus, the following groups of mice were formed: group with MS in early life and SS in adult life, group with only SS in adult life and control group, which was not exposed to any type of stress. For estimation of susceptibility to social stress we evaluated levels of anxiety in elevated plus-maze test, exploratory, communicative and locomotor activity in social interactions test and depressive-like behavior in forced swim test.

Results: Group of mice with MS in early life and SS in adult life demonstrated more depressive-like traits in forced swim test: they had significantly decreased time of the first immobility in comparison with group subjected to only SS in adult life. All experimental groups exposed to social stress both maternal separated in early life, and without such separation, showed significant behavioral alterations in comparison with control group. Thus, they demonstrated less time spent in open arms and significantly increased time spent in closed arms in elevated plus-maze test, which indicated increased level of anxiety in comparison with control group. Moreover, all groups with SS showed decreased moved distance and communicative features in social interactions test compared with control group.

Conclusion: Repeated maternal separation in early life leads to increased sensibility for social stress in adult life. Thus, early life stress is a potential risk factor for development of various mood diseases.

Acknowledgements: Supported by the RFBR (18-34-00603). 